EPJ Web of Conferences 75, 03008 (2014)

DOI: $10.1051 /$ epjconf/ 20147503008

(c) Owned by the authors, published by EDP Sciences, 2014

\title{
Growth of $\mathrm{CO}_{2} \mathrm{MnAl}$ Thin Films by Pulsed Laser Deposition
}

\author{
Samih Isber ${ }^{1, a}$, Nibelle Skaff ${ }^{1}$, Mohamad Roumie $^{2}$ and Malek Tabbal ${ }^{1}$ \\ ${ }^{1}$ American University of Beirut, Department of Physics, Bliss Street, Riad el Solh 11072020 Beirut, Lebanon \\ ${ }^{2}$ Lebanese Atomic Energy Commission-CNRS, Airport Rd., Riad El Solh 1107 2260, Beirut, Lebanon.
}

\begin{abstract}
The effects of the deposition temperature and laser energy on the characteristics of $\mathrm{Co}_{2} \mathrm{MnAl}$ films deposited on GaAs substrates were investigated. The grown films were characterized by AFM for film roughness and surface topography. Film thickness and elemental composition were measured using Rutherford Back Scattering (RBS) technique, while crystalline structure and phase composition were investigated by XRD. The RBS measurements showed that the stochiometry of the films was satisfactory and very close to that of the target Co: $0.5, \mathrm{Mn}: 0.25, \mathrm{Al}: 0.25$. The thickness of the films was found to increase as the laser energy was increased from 200 to $400 \mathrm{~mJ}$, in particular for the films deposited at $400{ }^{\circ} \mathrm{C}$. We also found an increase in the films thicknesses as the deposition temperature was increased for the samples grown at 200 and $300 \mathrm{~mJ}$. The best film quality as deduced from XRD, RBS and AFM results for producing these single layers were those deposited at $600{ }^{\circ} \mathrm{C}$ with the laser energy at $300 \mathrm{~mJ}$.
\end{abstract}

\section{Introduction}

Cobalt based Half metallic ferromagnets (HMF) have a band gap for minority electrons at the Fermi level, so that only one spin state is occupied at $\mathrm{T}=0 \mathrm{~K}$ [1]. These materials acquire remarkable properties for which the conduction is metallic only for one direction of electron spin and insulating for the other spin direction. Heusler alloys (such as $\mathrm{Co}_{2} \mathrm{MnAl}$, and $\mathrm{Ni}_{2} \mathrm{MnGa}$ ) constitute an important class of HMF among several other types such as iron oxide $\left(\mathrm{Fe}_{3} \mathrm{O}_{4}\right)$, rutile $\left(\mathrm{CrO}_{2}\right)$; as well as dilute magnetic semiconductors (ZnMnSe, GaMnAs). Since their discovery, Heusler ferromagnets have received considerable attention due to better control of stochiometry and more diverse magnetic and structural phenomena. Furthermore, on the technological level, Heusler ferromagnets are suitable candidates for room temperature devices due to their high degree of spin polarization, their structure that matches most of zincblende semiconductors, and their high ferromagnetic transition temperature $\left(T_{c}>600 \mathrm{~K}\right)[2-4]$. The synthesis and characterization of half-metal based compounds thin films represent an interesting and challenging task for spintronics devices [5,6]. Pulsed Laser Deposition (PLD) is a highly flexible and conceptually simple technique that offers the possibility of synthesizing novel materials under conditions that are far from thermal equilibrium [7-11]. In this work, we are proposing to investigate the synthesis of Heusler ferromagnet alloys $\left(\mathrm{Co}_{2} \mathrm{MnAl}\right)$ thin films by the PLD technique. Our goal is to investigate the structural and stoichiometric properties of the Heusler materials grown by PLD to optimize the growth conditions as function of

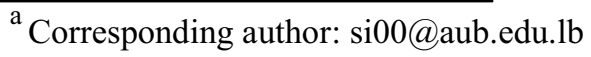

temperature deposition and laser energy.

\section{Experimental arrangements}

$\mathrm{Co}_{2} \mathrm{MnAl}$ thin films were grown on GaAs (100) substrates using a "SURFACE" PLD system consisting of a $248 \mathrm{~nm}$ wavelength $\mathrm{KrF}$ excimer laser with $20 \mathrm{~ns}$ pulse duration and a repetition rate ranging of $5 \mathrm{~Hz}$. High purity polycrystalline $\mathrm{Co}_{2} \mathrm{MnAl}$ target (MaTeck $\mathrm{GmbH}$ ) was used in all our deposition runs, the deposition time for all films was set to 20 minutes. During the growth, the target was set to rotate and toggle so that its surface remains homogenous with no drilling, thereby avoiding any plume disorientation from the substrate. The GaAs substrate was put at around $5 \mathrm{~cm}$ above the target. The deposition temperature was measured using a thermocouple placed on the back of the substrate. During the deposition of the films the chamber residual pressure was below $10^{-5} \mathrm{~Pa}$. Our study focused on the effect of deposition temperature and laser energy on the various properties of the grown films. Films were grown at substrate temperatures $\mathrm{T}_{\mathrm{s}}=400^{\circ} \mathrm{C}, 500^{\circ} \mathrm{C}$, and $600^{\circ} \mathrm{C}$ and at each temperature we deposited three films at laser energy of 200 , 300 and $400 \mathrm{~mJ}$. The nine deposited films were characterized by three different techniques. (i) The crystal structure of the films was investigated by Grazing Incidence X-Ray Diffraction (GIXRD). (ii) The surface morphology of the films were investigated and Atomic Force Microscopy (AFM). (iii) The elemental composition of the films and their thicknesses were determined using Rutherford Backscattering Spectrometry (RBS). The GIXRD measurements were performed using a fully automated 
diffractometer from Bruker AXS systems, using a $\mathrm{Cu} \mathrm{K}{ }_{\alpha} \mathrm{X}$ ray source emitting at $0.15418 \mathrm{~nm}$. The diffraction patterns were obtained by scanning the detector from $2 \theta=0^{\circ}$ to $80^{\circ}$, while setting the incidence angle at $2^{\circ}$. The analysis of the spectra were carried out using the EVA software package from Bruker that contains a search and match option to identify various phases of the films. A MultiMode ${ }^{\mathrm{TM}}$ Scanning Probe Microscope (NanoScope III) from Digital Instruments was used to obtain AFM images of the surface morphology of the films. All measurements were performed under contact mode using a silicon nitride cantilever, integrated with a sharp tip that is approximated by a pyramid formed from intersected [111] silicon planes. The scan size ranged from 1 to $10 \mu \mathrm{m}$, and the surface topography of all films was quantified through the determination of the lateral grain size, and root-mean-square surface roughness. The thickness and elemental composition of the films were determined by RBS; a Van de Graff tandem accelerator was used to accelerate a beam of ${ }^{4} \mathrm{He}^{2+}$ ions to $2 \mathrm{MeV}$, which was focused to hit the surface with a 1 $\mathrm{mm}^{2}$ spot size. The backscattered ions were detected at a scattering angle of $165^{\circ}$. The obtained RBS spectra were simulated using the SIMNRA software. The obtained values of film thickness were compared to those measured using an Ambios XP-1 high resolution surface profiler.

\section{Results and discussion}

Fig. 1 shows the GIXRD patterns of films grown under low background pressure $\left(\sim 10^{-5} \mathrm{~Pa}\right)$ and at various substrate temperatures $\left(\mathrm{T}_{\mathrm{s}}\right)$. The GIXRD spectra of the grown films showed that the films are single phase poly-crystalline $\mathrm{Co}_{2} \mathrm{MnAl}$.

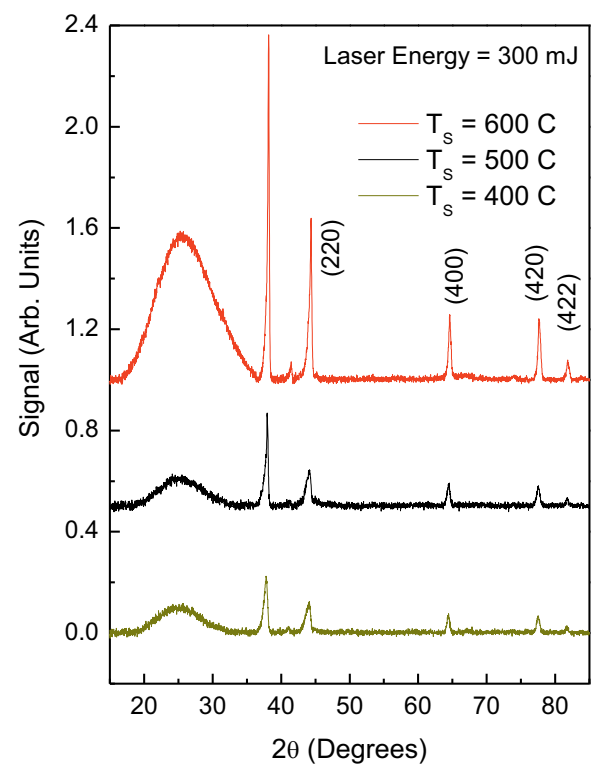

Figure 1. GIXRD peaks of $\mathrm{Co}_{2} \mathrm{MnAl}$ films at laser energy of $300 \mathrm{~mJ}$ and substrate temperatures 400,500 and $600^{\circ} \mathrm{C}$.

Furthermore, the XRD peaks assignment shows that $\mathrm{Co}_{2} \mathrm{MnAl}$ crystalline size is improved as the substrate temperature is increased. Indeed, the Full Width at Half Maximum (FWHM) of the X-ray peaks revealed a slight decrease with the increase in deposition temperature. The film thickness was measured using both RBS and the surface profilometry. We noticed slight difference between the thickness values found from the profiler and those obtained by RBS, in some cases this difference is about $30 \%$. However, for most samples this difference was below $10 \%$.

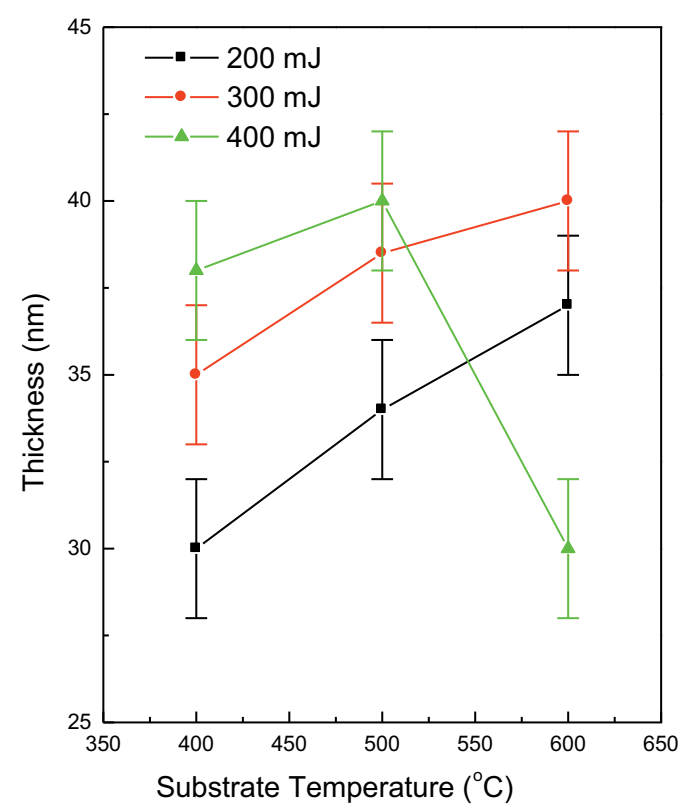

Figure 2. RBS spectrum of $\mathrm{Co}_{2} \mathrm{MnAl}$ thin film grown at laser energy of $300 \mathrm{~mJ}$ and substrate temperatures $500{ }^{\circ} \mathrm{C}$.

The RBS spectrum of $\mathrm{Co}_{2} \mathrm{MnAl} / \mathrm{GaAs}$ film deposited at a substrate temperature of $500{ }^{\circ} \mathrm{C}$ and laser energy of $300 \mathrm{~mJ}$ is shown, with its simulated fit, in Fig. 2. The thickness of the film as deduced from the fitting of the spectrum was found to be $40.5 \mathrm{~nm}$ compared to $38 \mathrm{~nm}$ as deduced from the profilometer measurement. The stoichiometric composition of this film was found to be $58 \%, 22 \%$ and $20 \%$ atomic for $\mathrm{Co}, \mathrm{Mn}$ and $\mathrm{Al}$, respectively. These values were obtained by simulating the RBS spectrum using the SIMNRA software. The effects of substrate temperature on film thickness, for the three values of the Laser energy are shown in Fig. 3. There is a clear trend that deposition at higher substrate temperature and laser energy leads to thicker films with the exception of the film deposited at $600^{\circ} \mathrm{C}$ and laser energy $400 \mathrm{~mJ}$. The surface morphology of the grown films was investigated by AFM measurements. It was noticed that as the substrate temperature is increased from $400{ }^{\circ} \mathrm{C}$ to $500{ }^{\circ} \mathrm{C}$ at constant laser energy, the roughness of the films is increased. However, for films deposition at substrate temperature of $600{ }^{\circ} \mathrm{C}$, the roughness did not change much as compared to those deposited at 500 ${ }^{\mathbf{0}} \mathrm{C}$. On the other hand, as we increase the laser energy at constant deposition temperature, the roughness increases. We can conclude that for a given laser energy, it is best to use a deposition temperature at about $600{ }^{\circ} \mathrm{C}$ to have smoother films. In our case, it was found that the sample grown at $600^{\circ} \mathrm{C}$ and $300 \mathrm{~mJ}$ produce relatively smooth films with average roughness of about $0.5 \mathrm{~nm}$, and an average grain size of $80 \mathrm{~nm}$. 


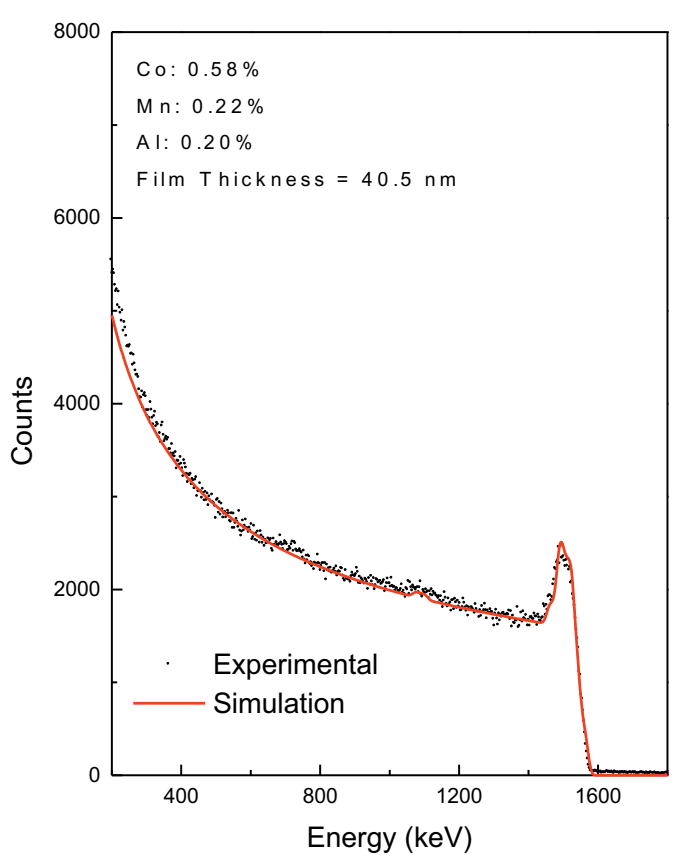

Figure 3. Films thickness vs substrate temperatures for various laser energies.

\section{Conclusion}

We have investigated the growth of $\mathrm{Co}_{2} \mathrm{MnAl}$ thin films on GaAs by laser ablation at substrate temperature of 400,500 and $600{ }^{\circ} \mathrm{C}$. The substrate temperature proved to be the key parameter in obtaining good quality films. It was found that at higher temperatures, films become more crystalline with larger grain size. The best film quality as deduced from XRD, RBS and AFM results for producing these single layers was obtained for substrate temperature of $600{ }^{\circ} \mathrm{C}$ and laser energy of $300 \mathrm{~mJ}$.

\section{Acknowledgment:}

This work was supported by the University Research Board (URB) of AUB, the Lebanese National Council for Scientific Research (LNCSR) and the Central Research Science Laboratory (CRSL) of the American University of Beirut.

\section{References}

1. G.A. Prinz, Phys. Rev. Lett. 54, 1051 (1985)

2. Y. Chen, D. Basiaga, J.R. O'Brien, and D. Heiman, Appl. Pys. Lett. 84, 4301 (2004)

3. J. W. Dong, L. C. Chen, C. J. Palmstrom, R. D. James, and S. McKernan, Appl. Phys. Lett. 75, 1443 (1999)

4. T. Ambrose, J. J. Krebs, and G. A. Prinz, Appl. Phys. Lett. 76, 3280 (2000)

5. G. A. Prinz, Science 282, 1660 (1998)

6. S. A. Wolf, D. D. Awschalom, R. A. Buhrman, J. M. Daughton, and S. von Molnar, Science 294, 1488 (2001)

7. I. Žutić, J. Fabian, and S. Das Sarma, Rev. Mod. Phys. 76, 323 (2004)
8. S. Isber, Y. J. Park, J. S. Moodera, and D. Heiman, J. Appl. Phys. 103, 07D713 (2008)

9. S. Gardelis, J. Androulakis, O. Monnereau, P. D. Buckle and J. Giapintzakis, Journal of Physics: Conference Series 10, 167 (2005)

10. X. Y. Dong, C. Adelmann, J. Q. Xie, C. J. Palmstrøm, X. Lou, J. Strand, P. A. Crowell J. P. Barnes and A. K. Petford-Long, Appl. Phys. Lett. 86, 102107 (2005)

11. W. H. Wang, M. Przybylski, W. Kuch, L. I. Chelaru, J. Wang,Y.F. Lu, J. Barthel and J. Kirschner, J. Magn. Magn. Mater. 286, 336 (2005) 